ISSN 0258-7122

Bangladesh J. Agril. Res. 34(2) : 269-278, June 2009

\title{
IN VITRO PROPAGATION OF BANANA
}

\author{
M REZAUL KARIM ${ }^{1}$, M. A. MALEK ${ }^{2}$, SAJIA RAHMAN ${ }^{3}$ \\ M. AL-AMIN ${ }^{4}$ AND M. RUHUL AMIN ${ }^{5}$
}

\begin{abstract}
An in vitro technique for plant regeneration using meristem-derived plantlets of banana cv. BARI-l (Musa sp.) has been developed. Highest number of shoot regeneration was noticed on basal media supplemented with $7.5 \mathrm{mgL}^{-1} \mathrm{BAP}+$ $0.5 \mathrm{mgL}^{-1} \mathrm{NAA}$ at 30 days after inoculation (DAI). The mean number of shoots significantly reduced when the concentrations of BAP and NAA in the medium was high. Regenerated shoots were rooted on half strength MS medium containing $0.5 \mathrm{mgL}^{-1}$ IAA $+0.5 \mathrm{mgL}^{-1}$ IBA at 30 DAI. In vitro raised plantlets were transferred to poly bags containing ground soil and cowdung mixture (1:1) for acclimatization and hardening in room temperature $\left(28-30^{\circ} \mathrm{C}\right)$ and the established plantlets are ready for planting in the field.
\end{abstract}

Key Words: In vitro propagation, banana (Musa sp.).

\section{Introduction}

The banana and plantains (Musa spp.) belonging to the family Musaceae is one of the world's most important subsistence crops. It is originated in Malaysia through a complex hybridization process (Novak, 1992). It is widely grown in the tropics and subtropic regions of the world. In Bangladesh, banana is popular for its year round availability, abundant production as well as high acceptability to the consumers. It is a rich source of carbohydrate and also rich in some minerals, notably phosphorus, calcium, and potassium. Banana is particularly rich in vitamin $\mathrm{C}$ and also contains significant amounts of several other vitamins (INIBAP, 1987). In addition, it has importance for tannin, latex and fiber production. Banana occupies an area of 45 thousand hectares of land with total production of 909 thousand metric tones with an average yield of $20.20 \mathrm{t} / \mathrm{ha}$ (BBS, 2006). This yield is quite low compared to other banana growing countries of the world like Argentina (34 t/ha) and Costa Rica (33 t/ha) (FAO, 2002). The low yield and production of banana is influenced by many biotic and abiotic stresses. Among the biotic stress, virus disease is one of the important factors. The traditional clonal propagation method appears unable to satisfy the increase in demand for disease free and healthy planting materials of banana. The productivity of vegetative propagated crops, such as banana and plantain is greatly reduced by virus disease (Lepoivre, 2000).

${ }^{1 \& 4}$ Biotechnology Divn., BARI, Joydebpur, Gazipur, ${ }^{2 \& 3}$ Plant Genetic Resources Centre, BARI, Joydebpur, Gazipur, $1701{ }^{5}$ Shere-Bangla Agricultural University, Dhaka- 1207, Bangladesh. 
Meristem culture offers an efficient method for rapid clonal propagation, production of virus free materials and germplasm preservation in plants (Cronauer and Krikorian, 1984a; Hwang et al., 2000 and Helloit et al., 2002). As regards yield performance, tissue cultured plants have been reported to produce 39\% higher yield than plants from sword suckers (Pradeep et al., 1992). Under Bangladesh conditions, tissue culture derived plantlets of banana performed better than the conventional sword suckers (Faisal et al., 1998). So, the present work was conducted at the laboratory of Biotechnology Division, Bangladesh Agricultural Research Institute (BARI), Gazipur.

\section{Materials and Method}

The plant materials of banana cv. BARI-l were collected from Biotechnology Division, Bangladesh Agricultural Research Institute (BARI), Joydebpur, Gazipur. The meristem used for establishment of culture was prepared under the microscope from the collected suckers through dissection and removal of leaf sheath. The meristem was obtained from developing suckers (about four months of age) of banana cv. BARI- 1 grown under field conditions and was brought to the preparation room. The suckers were washed thoroughly under running tap water. The roots and outer tissues of the suckers were removed with the help of a sharp knife. A number of outer leaves were removed until the shoot measured about 1.0-2.0 $\mathrm{cm}$ in length and $1.0 \mathrm{~cm}$ width at the base. The initial explant was prepared under stereomicroscope by removal of outer tissue of meristem with the help of sterile scalpel, which was about $5 \times 5 \mathrm{~mm}$ in size and the excised explants were surface sterilized with $0.1 \% \mathrm{HgCl}_{2}$ for 15 minutes and then placed on MS media supplemented with different concentrations of $\operatorname{BAP}(0.0,2.5,5.0,7.5$, and $\left.10.0 \mathrm{mgL}^{-1}\right)$ and NAA $\left(0.0,0.5,1.0,1.5\right.$, and $\left.2.0 \mathrm{mgL}^{-1}\right)$ for in vitro shoot regeneration. The culture tubes with media were autoclaved at $1.06 \mathrm{~kg} / \mathrm{cm}^{2}$ pressure at $121^{\circ} \mathrm{C}$ for 25 minutes. The medium was then cooled at room temperature before use. Sterilization of media was done after adding hormone.

\section{Results and Discussion}

\section{Regeneration of shoot from meristem explants}

In vitro culture of meristem (Plate 3 ) showed hard meristematic ball like structure in regeneration media containing different concentrations of BAP and NAA (Plate 4). The culture meristems first turned brown in colour in 4-5 days and after 30-50 days a green globular hard coat mass grow round in shape producing a ball like structure. From this ball like structure, adventitious plantlets were developed (Plate 5). Rahaman et al. (2004) observed that hard ball like structure developed from meristem explant in MS media supplemented with $5.0 \mathrm{mgL}^{-1}$ BAP. They also noticed that single shoot regeneration from meristem explant was thinner than shoot derived from shoot tip. Rabbani et al. (1996) denoted that 2ip have the 
more effective in producing ball like structure. Similar results were also obtained by Habib (1994) and Ali (1996) in their experiments. They observed that some ball like structures formed from the base of the shoot during shoot multiplication. These balls like structures are suitable for in vitro germplasm conservation.

Plant regeneration and subsequent shoot multiplication from meristem derived plantlets of banana cv. BARI-1 were observed in MS medium supplemented with different concentrations of BAP and NAA. The results obtained from this study are presented in Table 1-4 and discussed under the following sub-heads:

Table 1. Effect of different concentrations of BAP and NAA on shoot multiplication of banana at different days after inoculation.

\begin{tabular}{|c|c|c|c|c|c|c|c|}
\hline \multicolumn{2}{|c|}{ Treatments } & \multicolumn{3}{|c|}{ Shoot number } & \multicolumn{3}{|c|}{ Shoot length $(\mathrm{cm})$} \\
\hline $\begin{array}{c}\mathrm{BAP} \\
\left(\mathrm{mgL}^{-1}\right)\end{array}$ & $\begin{array}{c}\text { NAA } \\
\left(\mathrm{mgL}^{-1}\right)\end{array}$ & $10 \mathrm{DAI}$ & $20 \mathrm{DAI}$ & 30 DAI & $10 \mathrm{DAI}$ & 20 DAI & $30 \mathrm{DAI}$ \\
\hline \multirow{5}{*}{0} & 0 & $0.0 \mathrm{~b}$ & $1.00 \mathrm{c}$ & $1.00 \mathrm{f}$ & $0.00 \mathrm{i}$ & $0.00 \mathrm{j}$ & $0.00 \mathrm{j}$ \\
\hline & 0.5 & 1.0a & $1.50 \mathrm{bc}$ & 2.25def & $0.30 \mathrm{gh}$ & 1.03ghi & 1.88defgh \\
\hline & 1.0 & $0.5 \mathrm{ab}$ & $1.25 \mathrm{bc}$ & $2.50 \mathrm{de}$ & $0.30 \mathrm{gh}$ & 1.28 defgh & 1.95 bcdefg \\
\hline & 1.5 & $1.0 \mathrm{a}$ & $1.50 \mathrm{bc}$ & 1.50 ef & $0.23 \mathrm{~h}$ & 1.68 bcde & $2.33 \mathrm{~b}$ \\
\hline & 2.0 & $0.75 \mathrm{a}$ & 1.25 bc & 2.00 def & $0.20 \mathrm{~h}$ & 1.40 cdefg & $1.78 \mathrm{fgh}$ \\
\hline \multirow{5}{*}{2.5} & 0 & $1.0 \mathrm{a}$ & $1.00 \mathrm{c}$ & 1.50ef & $0.23 \mathrm{~h}$ & 1.23 & 1.9ocdefg \\
\hline & 0.5 & $1.0 \mathrm{a}$ & $1.25 \mathrm{bc}$ & $1.75 \mathrm{ef}$ & $0.30 \mathrm{gh}$ & 1.48 cdefg & 2.08 bcdefg \\
\hline & $1.0 \mathrm{a}$ & $1.0 \mathrm{a}$ & $1.25 \mathrm{bc}$ & $2.50 \mathrm{de}$ & 0.45 feg & 1.28 defgh & 1.80 efgh \\
\hline & 1.5 & 1.0a & 1.75abc & $1.75 \mathrm{ef}$ & 0.35 fgh & 1.lofghi & 1.83efgh \\
\hline & 2.0 & $1.0 \mathrm{a}$ & $1.50 \mathrm{bc}$ & 2.25def & $0.45 \mathrm{efg}$ & $0.70 \mathrm{i}$ & 1.50hi \\
\hline \multirow{5}{*}{5.0} & 0 & 1.0a & $1.25 b c$ & $1.50 \mathrm{ef}$ & $0.25 \mathrm{~h}$ & $0.88 \mathrm{hi}$ & $2.28 \mathrm{bc}$ \\
\hline & 0.5 & $1.0 \mathrm{a}$ & $1.75 \mathrm{a}$ & $2.50 \mathrm{de}$ & $0.48 \mathrm{ef}$ & $2.43 \mathrm{a}$ & 3.13 a \\
\hline & 1.0 & $1.0 \mathrm{a}$ & $2.25 \mathrm{ab}$ & 2.50de & $0.88 \mathrm{abc}$ & 2.05ab & 2.13bcdef \\
\hline & 1.5 & $1.0 \mathrm{a}$ & $1.50 \mathrm{bc}$ & $2.50 \mathrm{de}$ & $0.58 \mathrm{de}$ & $1.53 \mathrm{cdef}$ & $1.78 \mathrm{fgh}$ \\
\hline & 2.0 & $0.75 a$ & $1.50 \mathrm{bc}$ & $1.75 \mathrm{ef}$ & $0.55 \mathrm{e}$ & 1.28defgh & $1.70 \mathrm{gh}$ \\
\hline \multirow{5}{*}{7.5} & 0 & $0.75 \mathrm{a}$ & $1.75 \mathrm{abc}$ & $2.50 \mathrm{de}$ & $0.58 \mathrm{de}$ & 1.20 efgh & $1.25 \mathrm{i}$ \\
\hline & 0.5 & $0.75 \mathrm{a}$ & $2.75 \mathrm{a}$ & 6.25 a & $1.03 \mathrm{a}$ & $2.45 \mathrm{a}$ & $3.38 \mathrm{a}$ \\
\hline & 1.0 & $0.75 \mathrm{a}$ & $2.75 \mathrm{a}$ & $5.25 \mathrm{ab}$ & $0.95 \mathrm{ab}$ & $1.75 \mathrm{bcd}$ & $2.23 \mathrm{bcd}$ \\
\hline & 1.5 & 1.0a & $1.75 \mathrm{abc}$ & $4.25 b c$ & $0.88 \mathrm{abc}$ & $1.88 \mathrm{bc}$ & 2.10bcdef \\
\hline & 2.0 & 1.0a & $1.75 \mathrm{abc}$ & 2.25 def & $0.78 \mathrm{c}$ & 1.3odefgh & 2.05 bcdefg \\
\hline \multirow{5}{*}{10.0} & 0 & $1.0 \mathrm{a}$ & $1.25 b c$ & 2.25def & $0.75 c$ & 1.05 fghi & 1.93 cdefg \\
\hline & 0.5 & $0.75 a$ & 2.00abc & 2.50de & $0.73 \mathrm{~cd}$ & 1.60bcde & 2.18bcde \\
\hline & 1.0 & $0.75 \mathrm{a}$ & $2.00 \mathrm{abc}$ & $2.50 \mathrm{de}$ & $0.80 \mathrm{bc}$ & 1.48 cdefg & 2.28 bc \\
\hline & 1.5 & $0.5 \mathrm{ab}$ & $1.50 \mathrm{bc}$ & $3.25 \mathrm{~cd}$ & $0.73 \mathrm{~cd}$ & 1.35defgh & 1.98bcdefg \\
\hline & 2.0 & $0.75 \mathrm{a}$ & $1.50 \mathrm{bc}$ & $2.50 \mathrm{de}$ & $0.73 \mathrm{~cd}$ & 1.23 efgh & 1.90 cdefg \\
\hline \multirow{2}{*}{\multicolumn{2}{|c|}{$\begin{array}{l}\text { LSD value (0.Ol) } \\
\text { CV (\%) }\end{array}$}} & 0.61 & 1.05 & 1.26 & 0.16 & 050 & 0.39 \\
\hline & & 38.88 & 34.55 & 26.71 & 16.06 & 19.27 & 10.49 \\
\hline
\end{tabular}




\section{Shoot number and shoot length per explant}

The number of shoots produced per explant varied in MS media supplemented with different concentrations of BAP and NAA. Data were recorded at 10, 20, and 30 days after inoculation (DAI) and results are presented in Table 1 . The effect of different concentrations of BAP and NAA on shoot regeneration and proliferation were statistically significant at $1 \%$ level of significance. Among the different concentrations, $7.5 \mathrm{mgL}^{-1} \mathrm{BAP}+0.5 \mathrm{mgL}^{-1} \mathrm{NAA}$ showed highest shoot proliferation $(0.75,2.75$, and 6.25 shoots per explant) at 10,20 , and $30 \mathrm{DAI}$, respectively. A good number of shoot proliferations was achieved at $7.5 \mathrm{mgL}^{-1}$ BAP $+1.5 \mathrm{mgL}^{-1} \mathrm{NAA}$ at $30 \mathrm{DAI}$ (4.25), which is superior to the control treatments (1.00). The regeneration and proliferation of shoots was sequentially described in plate $6,7,8$, and 9 from initial sub culture to $4^{\text {th }}$ sub culture. Rahaman et al. (2004) found highest shoot number at $1.5 \mathrm{mgL}^{-1} \mathrm{BAP}+\mathrm{NAA}$ (4.52 explant) at 30 DAI. The result of current investigation is not fully supported by Rabbani et al. (1996) where they found highest number of shoots per explant at 28 DAI $(3.11 \pm 0.66)$ with $5.0 \mathrm{mgL}^{-1}$ of BAP and $\mathrm{Kn}$. This variation might be due to the different concentrations of NAA and BAP and their associations.

The MS medium supplemented with BAP and NAA showed different results for increasing shoot length which was significantly influenced by different concentrations. The longest shoot was produced by the concentration of $7.5 \mathrm{mgL}^{-}$

${ }^{1} \mathrm{BAP}+0.5 \mathrm{mgL}^{-1} \mathrm{NAA}(1.03,2.45$ and $3.38 \mathrm{~cm})$ at 10, 20, and $30 \mathrm{DAI}$, respectively. Statistically identical shoot length was observed in 5.0 mgL' BAP + $0.5 \mathrm{mgL}^{-1} \mathrm{NAA}$ at $20 \mathrm{DAI}(2.43 \mathrm{~cm})$ and $30 \mathrm{DAI}(3.13 \mathrm{~cm})$. Rahaman et al. (2004) observed the similar result. They obtained longest shoot in $5.0 \mathrm{mgL}^{-1} \mathrm{BAP}$ $(3.62 \mathrm{~cm})$ using BARI Banana-1. They also found shortest leaf in $2.0 \mathrm{mgL}^{-1}$ BAP. The results of the present study agree with the findings of Khanam et al. (1996) who obtained longest shoot in banana on MS medium supplemented with $25 \mu \mathrm{M}$ BAP treatments.

\section{Leaf number and leaf length per explant}

The effect of different concentrations of NAA and BAP on leaf number and leaf length were presented in Table 2. The results showed that the maximum number of leaves (2.50, 3.25, and 7.00 leaves per explant at 10, 20, and $30 \mathrm{DAI})$ produced on the medium supplemented with $7.5 \mathrm{mgL}^{-1} \mathrm{BAP}$ and $0.50 \mathrm{mgL}^{-1}$ NAA. The second highest number of leaves (2.75, 4.00, and 6.75 leaves/explant at 10, 20, and $30 \mathrm{DAI}$, produced on the medium supplemented with $5.0 \mathrm{rngL}^{-1}$ BAP and $1.0 \mathrm{mgL}^{-1}$ NAA. Rahman et al. (2004) found the maximum number of leaves (3.12/plantlet) at 30 DAI produced with $5.0 \mathrm{mgL}^{-1}$ BAP, which was identical with the treatment of $4.0 \mathrm{mgL}^{-1} \mathrm{BAP}+1.50 \mathrm{mgL}^{-1} \mathrm{NAA}$. Rabbani et al. 
(1996) obtained same results from $5.0 \mathrm{mgL}^{-1} \mathrm{BAP}$. The lowest number of leaves $(0.00,0.50$, and 2.25 at 10,20 , and 30 DAI leaves per explant) were obtained from control treatment (Table 2).

Table 2. Effect of different concentrations of BAP and NAA on leaf number and leaf length of banana at different days after inoculation.

\begin{tabular}{|c|c|c|c|c|c|c|c|}
\hline \multicolumn{2}{|c|}{ Treatment } & \multicolumn{3}{|c|}{ Shoot number } & \multicolumn{3}{|c|}{ Shoot length (cm) } \\
\hline $\begin{array}{c}\text { BAP } \\
\left(\mathrm{mgL}^{-1}\right)\end{array}$ & $\begin{array}{c}\text { NAA } \\
\left(\mathrm{mgL}^{-1}\right)\end{array}$ & $10 \mathrm{DAI}$ & 20 DAI & $30 \mathrm{DAI}$ & $10 \mathrm{DAI}$ & 20 DAI & $30 \mathrm{DAI}$ \\
\hline \multirow{5}{*}{0} & 0 & $0.00 \mathrm{e}$ & $0.5 \mathrm{c}$ & $2.25 \mathrm{~d}$ & 0.50 defg & $0.55 \mathrm{~h}$ & $0.95 \mathrm{i}$ \\
\hline & 0.5 & $1.50 \mathrm{~cd}$ & $2.25 \mathrm{~b}$ & $4.75 \mathrm{c}$ & 0.58 cdef & $1.65 \mathrm{fg}$ & $2.03 \mathrm{gh}$ \\
\hline & 1.0 & $1.75 \mathrm{bcd}$ & $2.75 \mathrm{ab}$ & $4.50 \mathrm{c}$ & 0.60bcde & 1.70efg & $1.95 \mathrm{~h}$ \\
\hline & 1.5 & $1.75 \mathrm{bcd}$ & 3.00ab & $5.50 \mathrm{bc}$ & $0.70 \mathrm{abc}$ & 1.8obcdefg & $2.00 \mathrm{gb}$ \\
\hline & 2.0 & $1.50 \mathrm{~cd}$ & $2.25 b$ & $5.50 \mathrm{bc}$ & 0.46defg & $1.60 \mathrm{~g}$ & $2.08 \mathrm{gh}$ \\
\hline \multirow{5}{*}{2.5} & 0 & $1.50 \mathrm{~cd}$ & $2.50 \mathrm{~b}$ & $4.75 \mathrm{c}$ & 0.5odefg & $1.55 \mathrm{~g}$ & $1.95 \mathrm{~h}$ \\
\hline & 0.5 & $1.75 \mathrm{bcd}$ & $3.00 \mathrm{ab}$ & $5.00 \mathrm{c}$ & $0.63 \mathrm{bcd}$ & $2.03 \mathrm{bcd}$ & $2.58 \mathrm{efg}$ \\
\hline & 1.0 & $2.25 \mathrm{abc}$ & $3.25 \mathrm{ab}$ & $5.50 \mathrm{bc}$ & $0.600 \mathrm{bcdc}$ & 1.95 bcdef & 2.53 efgh \\
\hline & 1.5 & $1.50 \mathrm{~cd}$ & $2.75 \mathrm{ab}$ & 5.75abc & $0.45 \mathrm{efg}$ & $2.075 b c$ & $2.43 f g h$ \\
\hline & 2.0 & $2.00 \mathrm{abcd}$ & $3.25 \mathrm{ab}$ & $5.50 \mathrm{bc}$ & 0.45 efg & $2.05 \mathrm{bcd}$ & $2.28 \mathrm{gh}$ \\
\hline \multirow{5}{*}{5.0} & 0 & $1.25 \mathrm{~d}$ & $2.5 b$ & $4.75 \mathrm{c}$ & $0.35 \mathrm{~g}$ & $1.68 \mathrm{fg}$ & $2.15 \mathrm{gh}$ \\
\hline & 0.5 & $2,50 \mathrm{ab}$ & $3.25 \mathrm{ab}$ & $5.25 \mathrm{c}$ & $0.75 \mathrm{ab}$ & $2.60 \mathrm{a}$ & 3.60 bc \\
\hline & 1.0 & $2.75 \mathrm{a}$ & $4.00 \mathrm{a}$ & $6.75 \mathrm{ab}$ & $0.70 \mathrm{abc}$ & $2.05 \mathrm{bcd}$ & 3.10 cde \\
\hline & 1.5 & $2.25 \mathrm{abc}$ & $3.25 \mathrm{ab}$ & $5.50 \mathrm{bc}$ & 0.50 defg & $2.03 \mathrm{bcd}$ & 2.93 def \\
\hline & 2.0 & $1.75 \mathrm{bcd}$ & $2.75 \mathrm{ab}$ & $5.25 \mathrm{c}$ & 0.50 defg & 2.00 bcde & $2.98 \mathrm{def}$ \\
\hline \multirow{5}{*}{7.5} & 0 & $2.00 \mathrm{abcd}$ & $3.25 \mathrm{ab}$ & $5.25 \mathrm{c}$ & $0.43 \mathrm{fg}$ & $1.65 \mathrm{fg}$ & $2.23 \mathrm{gh}$ \\
\hline & 0.5 & $2.5 \mathrm{ab}$ & $3.25 \mathrm{ab}$ & $7.00 \mathrm{a}$ & 0.85 a & $2.70 \mathrm{a}$ & $4.23 \mathrm{a}$ \\
\hline & 1.0 & $1.75 \mathrm{bcd}$ & $2.75 \mathrm{ab}$ & 5.25 c & $0.70 \mathrm{abc}$ & $2.10 \mathrm{~b}$ & $3.70 \mathrm{ab}$ \\
\hline & 1.5 & 2.00abcd & $3.00 \mathrm{ab}$ & $5.75 \mathrm{abc}$ & 0. S5cdef & 1.95bcdef & 2.58efg \\
\hline & 2.0 & $2.00 \mathrm{abcd}$ & $2.5 \mathrm{~b}$ & $4.50 \mathrm{c}$ & 0.55 cdef & 1.76 cdefg & $2.28 \mathrm{gh}$ \\
\hline \multirow{5}{*}{10.0} & 0 & $1.50 \mathrm{~cd}$ & $3.25 a b$ & $4,75 c$ & 0.S3def & $1.53 g$ & $2.15 \mathrm{gh}$ \\
\hline & 0.5 & $2.00 \mathrm{abcd}$ & $2.5 \mathrm{~b}$ & $4.50 \mathrm{c}$ & 0.50 defg & 1.75 defg & 2.98 def \\
\hline & 1.0 & 1.50cd & $3.25 \mathrm{ab}$ & $5.25 c$ & $0.43 \mathrm{fg}$ & 1.93 bcdef & $3.23 \mathrm{bcd}$ \\
\hline & 1.5 & $2.25 \mathrm{abc}$ & $2.75 \mathrm{ab}$ & $4.75 \mathrm{c}$ & 0.45 efg & 1.95 bcdef & $3.23 \mathrm{bcd}$ \\
\hline & 2.0 & $1.75 \mathrm{bcd}$ & $2.75 \mathrm{ab}$ & $4.75 \mathrm{c}$ & 0.45 efg & $1.60 \mathrm{~g}$ & 3.08 cde \\
\hline \multicolumn{2}{|c|}{ L.SD value (0.01) } & 0.97 & 1.28 & 1.39 & 0.16 & 0.31 & 0.58 \\
\hline \multicolumn{2}{|l|}{ CV (\%) } & 28.71 & 24,22 & 14.46 & 15.12 & 8.86 & 11.86 \\
\hline
\end{tabular}


The MS medium supplemented with BAP and NAA showed different results for leaf length, which was significantly influenced by different concentrations. The longest leaves were produced by the concentration of $7.5 \mathrm{mgL}^{-1} \mathrm{BAP}+0.5$ $\mathrm{mgL}^{-1}$ NAA $(0.85,2.70$, and $4.23 \mathrm{~cm}$ at 10,20 , and 30 DAI, respectively, which was statistically significant. Statistically identical results was observed in 7.5 $\mathrm{mgL}^{-1} \mathrm{BAP}+1.0 \mathrm{mgL}^{-1} \mathrm{NAA}$ at 20 DAI $(2.10 \mathrm{~cm})$ and 30 DAI $(3.70 \mathrm{~cm})$. Rahaman et al. (2004) observed the similar result. They obtained longest leaf in the treatment $5.0 \mathrm{mgL}^{-1}$ BAP $(3.62 \mathrm{~cm})$ followed by $1.5 \mathrm{mgL}^{-1} \mathrm{NAA}$ and 4.0 $\mathrm{mgL}^{-1}$ BAP $(3.40 \mathrm{~cm})$ using BARI Banana-I. They also found shortest leaf in 2.0 $\mathrm{mgL}^{-1}$ BAP. The results of present experiment agree with the findings of Khanam et al. (1996) who obtained longest leaves in banana on MS medium supplemented with $25 \mu \mathrm{M}$ BAP treatments.

Table 3. Effect of different concentrations of IAA and IBA on root number of multiple shoot of banana at different days after inoculation.

\begin{tabular}{|c|c|c|c|c|c|}
\hline \multicolumn{2}{|c|}{ Treatment } & \multirow{2}{*}{$\begin{array}{l}\text { Vigor of } \\
\text { regenerated } \\
\text { root }\end{array}$} & \multicolumn{3}{|c|}{ Root number } \\
\hline IAA $\left(\mathrm{mgL}^{-1}\right)$ & IBA $\left(\mathrm{mgL}^{-1}\right)$ & & $10 \mathrm{DAI}$ & $20 \mathrm{DAI}$ & 30DAI \\
\hline \multirow{4}{*}{0} & 0 & + & $0.00 \mathrm{e}$ & 0.00 e & $0.00 \mathrm{f}$ \\
\hline & 0.5 & + & $1.50 \mathrm{~cd}$ & $2.00 \mathrm{~d}$ & $3.25 \mathrm{e}$ \\
\hline & 1.0 & ++ & $2.25 \mathrm{bc}$ & $2.25 \mathrm{~d}$ & $3.50 \mathrm{de}$ \\
\hline & 1.5 & ++ & $2.25 \mathrm{bc}$ & $2.50 \mathrm{~cd}$ & $3.50 \mathrm{de}$ \\
\hline \multirow{4}{*}{0.5} & 0 & +++ & $2.75 \mathrm{ab}$ & $2.25 \mathrm{~d}$ & $3.25 \mathrm{e}$ \\
\hline & 0.5 & +++ & 3.50 a & $4.50 \mathrm{a}$ & $6.50 \mathrm{a}$ \\
\hline & 1.0 & ++ & $3.25 \mathrm{ab}$ & $3.50 \mathrm{abc}$ & $6.00 \mathrm{ab}$ \\
\hline & 1.5 & ++ & $3.25 \mathrm{ab}$ & $4.00 \mathrm{ab}$ & $5.00 \mathrm{bc}$ \\
\hline \multirow{4}{*}{1.0} & 0 & + & $1.00 \mathrm{de}$ & $2.50 \mathrm{~d}$ & $3.25 \mathrm{e}$ \\
\hline & 0.5 & + & $2.75 \mathrm{ab}$ & $2.75 \mathrm{~cd}$ & 3.75 de \\
\hline & 1.0 & ++ & 1.SOcd & 3.O0bcd & 4.O0cde \\
\hline & 1.5 & + & $1.25 \mathrm{~cd}$ & $3.50 \mathrm{abc}$ & $4.50 \mathrm{~cd}$ \\
\hline \multicolumn{2}{|c|}{ LSD value (0.01) } & & 1.03 & 1.16 & 1.16 \\
\hline \multicolumn{2}{|l|}{ CV(\%) } & & 25.36 & 22.19 & 15.51 \\
\hline
\end{tabular}

$+=$ Less vigorous growth, $++=$ Good growth and vigor, $+++=$ Best growth and vigor

\section{Root number per explants}

The effect of IAA and IBA on number of roots per explant produced by different combinations at 10, 20, and 30 DAI (Table 3 ) showed significant variation. The highest number of roots was produced by $0.5 \mathrm{mgL}^{-1} \mathrm{IAA}+0.5 \mathrm{mgL}^{-1}$ IBA (3.50, 4.50, and 6.50 per explant, respectively), which was statistically significant than 
other treatments (Table 5). The treatment $0.5 \mathrm{mgL}^{-1} \mathrm{IAA}+1.0 \mathrm{mgL}^{-1} \mathrm{IBA}$ produced 6.0 roots per explant at $30 \mathrm{DAI}$, but at $20 \mathrm{DAI}, 3.50$ roots was produced per explant. Vigorous roots of in vitro grown plantlet on MS media supplemented with $0.5 \mathrm{mgL}^{-1} \mathrm{IAA}+0.5 \mathrm{mgL}^{-1}$ IBA was shown in plate 10 (a \& b). The present results are similar with the findings of Gubbuk and Pekmezci (2001). Molla et al. (2004) obtained 8.28 roots per plantlet on $0.5 \mathrm{mgL}^{-1}$ IBA followed by 6.33 roots in $0.6 \mathrm{mgL}^{-1}$ BAP that was similar to the present work. The results were also similar with the findings of Khanam et al. (1996) but Rahman et al. (2004) obtained only 2.88 roots per explant at 30 DAI by $3.0 \mathrm{mgL}^{-1} \mathrm{NAA}$.

Table 4. Effect of different concentrations of IAA and IBA on root length of multiple shoot of banana at different days after inoculation.

\begin{tabular}{|c|c|c|c|c|}
\hline \multicolumn{2}{|c|}{ Treatment } & \multicolumn{3}{|c|}{ Root number } \\
\hline IAA $\left(\mathrm{mgL}^{-1}\right)$ & IBA $\left(\mathrm{mgL}^{-1}\right)$ & $10 \mathrm{DAI}$ & $20 \mathrm{DAI}$ & 30DAI \\
\hline \multirow{4}{*}{0} & 0 & $0.00 \mathrm{e}$ & $2.00 \mathrm{ef}$ & $2.00 \mathrm{f}$ \\
\hline & 0.5 & $1.08 \mathrm{~d}$ & $1.88 \mathrm{f}$ & $2.30 \mathrm{e}$ \\
\hline & 1.0 & $1.08 \mathrm{~d}$ & 2.3Ode & $3.15 \mathrm{~d}$ \\
\hline & 1.5 & $1.13 \mathrm{~d}$ & $2.45 \mathrm{~d}$ & $3.08 \mathrm{~d}$ \\
\hline \multirow{4}{*}{05} & 0 & $1.23 \mathrm{~d}$ & $1.60 \mathrm{f}$ & $2.08 \mathrm{e}$ \\
\hline & 0.5 & $2.93 \mathrm{a}$ & $4.63 \mathrm{a}$ & $5.88 \mathrm{a}$ \\
\hline & 1.0 & $2.55 \mathrm{ab}$ & $3.88 \mathrm{~b}$ & $4.83 \mathrm{~b}$ \\
\hline & 1.5 & 3.03 a & $3.85 \mathrm{~b}$ & $4.88 \mathrm{~b}$ \\
\hline \multirow{4}{*}{10} & 0 & $1.80 \mathrm{c}$ & 2.33 de & $3.45 \mathrm{~cd}$ \\
\hline & 0.5 & $1.80 \mathrm{c}$ & 2.35 de & $3.48 \mathrm{~cd}$ \\
\hline & 1.0 & 2.08bc & $3.15 c$ & $3.75 c$ \\
\hline & 1.5 & 2.1Sbc & $2.70 \mathrm{~d}$ & $3.70 \mathrm{c}$ \\
\hline \multicolumn{2}{|c|}{ LSD value (0.01) } & 0.49 & 0.42 & 0.55 \\
\hline \multicolumn{2}{|c|}{ CV(\%) } & 16.28 & 7.95 & 7.57 \\
\hline
\end{tabular}

\section{Root length per explant}

The length of roots developed by the plantlets was influenced considerably by different concentrations of IAA and IBA and the results were presented in Table 4. The result indicated that there was an increasing trend in root length at different DAI $(10,20$, and $30 \mathrm{DAI})$, which is significant at $1 \%$ level. The root length of plantlets after 30 DAI was remarkably highest by auxin, which was essential for successful root induction of banana and has been reported by Raut and Lokhand (1989). The highest length was observed at 10, 20, and 30 DAI in concentration $0.5 \mathrm{mgL}^{-1}$ IAA and IBA $(2.93,4.63$, and $5.88 \mathrm{~cm})$, which was 
statistically significant. The second highest result (3.03, 3.85, and $4.88 \mathrm{~cm}$ at 10 , 20, and $30 \mathrm{DAI}$ ) was observed with $0.5 \mathrm{mgL}^{-1} \mathrm{IAA}$ and $1.5 \mathrm{mgL}^{-1}$ IBA. A similar result was obtained by Molla et al. (2004) where they got 2.60-5.67 cm root length in $0.5 \mathrm{mgL}^{-1}$ IBA. Habiba (2002), Khanam et al. (1996) and Ali (1996) also got more or less same observation.

\section{Establishment of plantlet}

Meristem derived plantlets were transferred to poly bags containing ground soil:cow dung mixture (1:1) for acclimatization and hardening in room temperature $\left(28-30^{\circ} \mathrm{C}\right)$ and a good number of established plant $(95 \%)$ is ready for planting in the field.

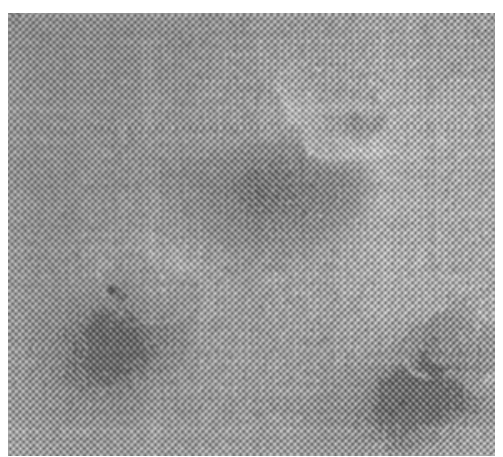

A. Excised meristem of banana cv. BARI-1.

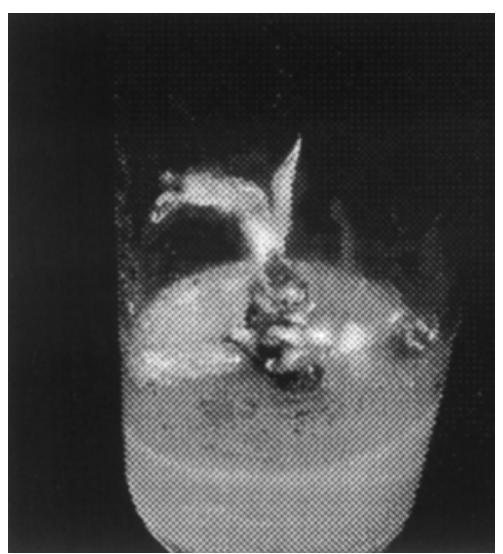

C. Multiple shoots with 7.5 $\mathrm{mgL}^{-1} \mathrm{BAP}+0.5 \mathrm{mgL}^{-1} \mathrm{NAA}$ at 30 days after inoculation.

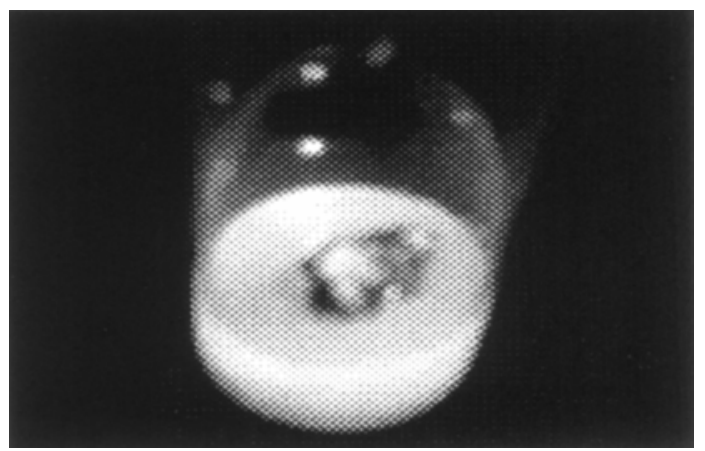

B. Shoot initiation from green globular hard coat meristematic ball like structure.

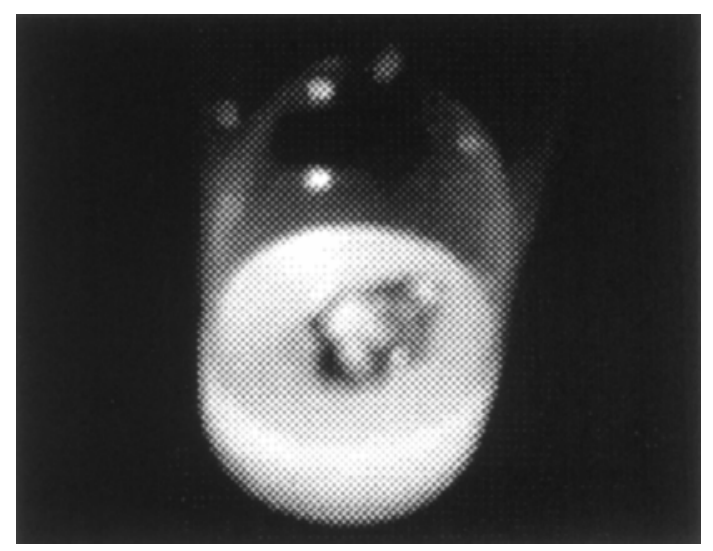

D. Multiple shoots with $7.5 \mathrm{mgL}^{-1} \mathrm{BAP}+0.5$ $\mathrm{mgL}^{-1} \mathrm{NAA}$ at $2^{\text {nd }}$ subculture. 


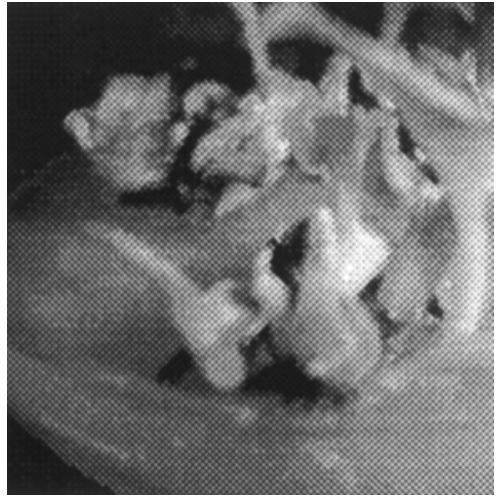

E. Multiple shoots with 7.5 $\mathrm{mgL}^{-1} \quad \mathrm{BAP}+0.5 \mathrm{mgL}^{-1}$ NAA at $3^{\text {rd }}$ subculture.

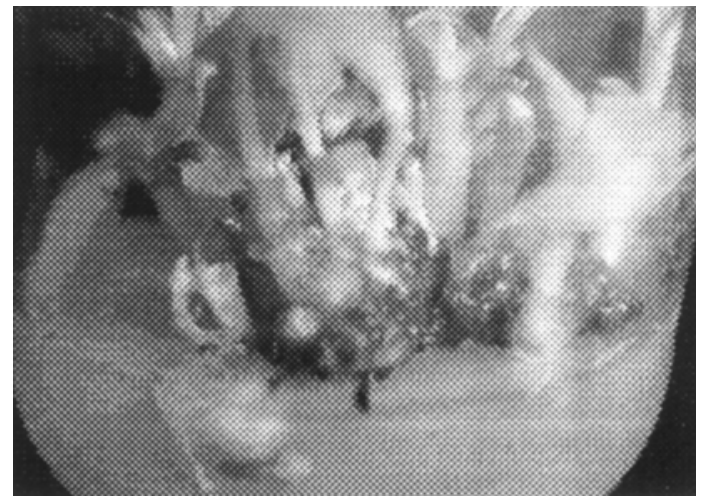

F. Multiple shoots with $7.5 \mathrm{mgL}^{-1} \mathrm{BAP}+0.5$ mgL $^{-1}$ NAA at $4^{\text {th }}$ subculture.

Fig. 1. Shoot regeneration from meristem derived of banana cv. BARI-1

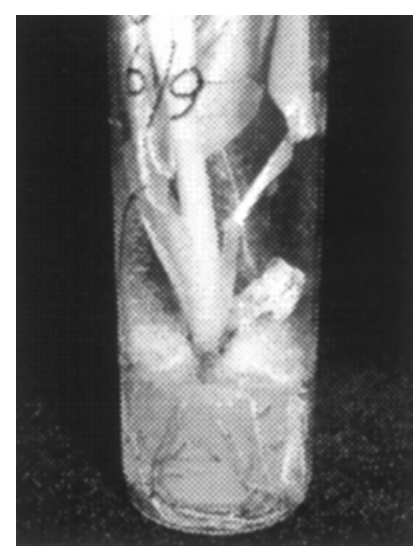

G. Vigorous roots with 0.5 $\mathrm{mgL}^{-1} \mathrm{IAA}+0.5 \mathrm{mgL}^{-1}$ IBA.

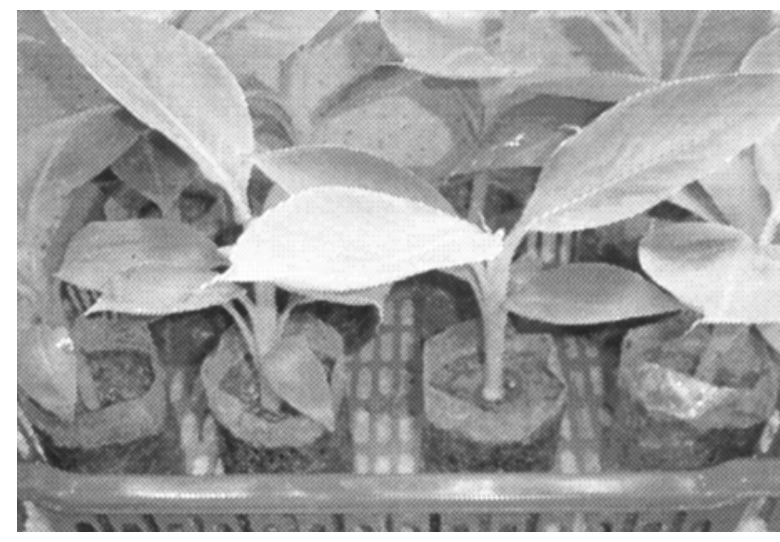

H. Well established meristern derived banana plants in poly bags.

Fig. 2. Plantlet developed from meristern derived banana plants in poly bags.

\section{References}

Ali, H. 1996. Effect of BAP and IBA on micropropagation of some banana cultivars. M.S. thesis, Department of Horticulture, Bangladesh Agricultural University, Mymensingh. $73 \mathrm{p}$.

BBS. 2006. Statistical Year Book of Bangladesh, Statistics Divn., Ministry of Planning, Govt. of the People s Republic of Bangladesh. p. 208.

Cronauer, S. S. and A. D. Krikorian. 1984a. Multiplication of Musa from excised stem tips. Ann. Bot., 53(3): 321-328. 
Faisal, S. M., M. A. Hoque and A. Quasem. 1998. Field performance of in vitro plantlets against normal suckers of banana (Musa sapientum) cv. Champa. Plant Tissue Cult. 8(2): 125-129.

FAO. 2002. Production Year Book 2000. Food and Agriculture Organization of the United Nations, Rome, Italy. Vol. 53, p 184.

Gubbuk, H. and M. Pekmezci. 2001. The effects of different hormone types and concentrations on propagation of different banana clones by meristem culture. Ziraat Fakultesi Dergisi, Akdeniz Universitesi 14(1): 127-137.

Habib, A. 1994. Mass propagation of Mussa sapientum var. Amritasagar and performance of different genotype of Mussa cavendish (Grand Naine) in Bangladesh. M. Sc. Thesis, Dept. of Botany, University of Dhaka. p. 53.

Habiba, U., S. Reja, M. L. Saha and M. R. Khan. 2002. Endogenous bacterial contamination during in vitro culture of table banana: Identification and prevention. Plant Tissue Cult. 12(2): 117-124.

Helliot, B., B. Panis, Y. Poumay, R. Swennen, P. Lepoivre and E. Frison. 2002. Cryopreservation for the elimination of cucumber mosaic and banana streak viruses from banana (Musa spp.). Plant Cell Rep. 20(12): 1117-1122.

Hwang Shinchuan, S. C. Hwang, A. B. Molina and V. N. Rao. 2000. Recent developments of Fusarium R \& D in Taiwan. Advancing banana and plantain R \& D in Asia and the Pacific. Proceeding of the 9th INIBAP-ASPNET. Regional Advisory Committee meeting held at South China Agricultural University, China, 2 5 November 1999. pp. 84-92.

INIBAP. 1987. International Network for the Improvement of Banana and Plantain, publication (October, 1987). Montpellier Cedex (France): pp. 8-9.

Khanam, D., M.A. Hoque, M.A. Khan and A. Quasem. 1996. In vitro propagation of banana (Musa spp). Plant Tiss. Cult. 6(2): 89-94.

Lepoivre, P. 2000. Banana in vitro regeneration: Virus eradication. Laboratory of plant pathology, University of Gembloux, Belgium, p. 22.

Molla, M.M.H., M. Dilafroza Khanam, M.M. Khatun, M. Al-Amin and M.A. Malek. 2004. In vitro rooting and Ex vitro plantlet establishment of BARI Banana (Musa sp.) as influenced by different concentrations of IBA (Indole 3-butyric Acid). Asian Jour. of Plant Sci. 3(2): 196-199.

Novak, F. J. 1992. Musa (Bananas and Plantains). In: Hammerschlag, F.A. and Litz., RE. (eds), Biotechnology of Perennial Fruit Crops. CAB International, University Press, Cambridge. U.K. pp. 449-488.

Pradeep, K. P., G. Zachariah, S. Estelittanad, A. Suma. 1992. Field performance of banana tissue culture plants of variety Nendran (Musa AAB). South Indian Hort. 40(1): 1-4.

Rabbani, M. G., M. H. Ali and M. F. Mondal. 1996. Effect of BAP and IBA on micropropagation of some banana cultivars. Bangladesh Hort. 25(1 \& 2): 47-52.

Rahman, M. Z., K.M. Nasiruddin, M. Al-Amin and M.N. Islam. 2004. In vitro response and shoot multiplication of banana with BAP and NAA Asian of Plant Sci. 3(4):406409.

Raut, R.S. and V.E. Lokhande. 1989. Propagation of plantain through meristem culture. Ann. Plant Physiol. 3(2): 256-260. 